\title{
Evaluación de complicaciones de la fístula arteriovenosa para hemodiálisis según la técnica de canalización
}

\author{
Ian Blanco Mavillard, Miguel Ángel Rodríguez Calero, Cynthia Sánchez Rojas \\ Hospital de Manacor. Universidad de las Islas Baleares. Mallorca. España
}

\section{Resumen}

Introducción: La fístula arteriovenosa es el acceso vascular de elección para los tratamientos en hemodiálisis, puncionado periódicamente tres veces por semana. Existen tres tipos de técnicas de punción: en escalera, en área de punción y buttonhole. Las técnicas convencionales son causantes de gran morbilidad a medio y largo plazo, sin embargo, el Buttonhole está asociado a priori a la mejoraría de esos problemas.

Objetivo: Comparar la técnica de punción de buttonhole con respecto a las técnicas convencionales para la canulación del acceso vascular en el tratamiento de Hemodiálisis, en función del grado de dolor asociado a la técnica, tasas de infección asociadas y la repercusión sobre la supervivencia del acceso vascular.

Material y Método: Revisión bibliográfica narrativa. Se incluyeron estudios experimentales y observacionales en inglés y castellano publicados entre 2010 y 2015 , que compararan la técnica de punción convencional respecto a la técnica de punción Buttonhole. Selección inicial por título y resumen. Se utilizaron las escalas CASPe y STROBE para la evaluación metodológica.

Resultados: Se identificaron 1.827 estudios, de los que seleccionamos 13 tras el proceso de evaluación crítica. La heterogeneidad de resultados no permite afirmar que ninguna de las técnicas analizadas mejore los resultados en dolor en la punción o supervivencia del acceso, sin embargo, se asocia un aumento de eventos infecciosos con la técnica de Buttonhole.

Correspondencia:

Ian Blanco Mavillard

Hospital de Manacor. Unidad de Calidad

Ctra. Manacor-Alcudia s/n. 07500 Manacor. Mallorca

E-mail: ianblanco7@gmail.com
Conclusión: no se han hallado argumentos para asegurar que el Buttonhole pueda ser considerado una técnica de primera elección, debido a la heterogeneidad de las formas de medida de los estudios revisados.

PALABRAS CLAVE: hemodiálisis; fistula arteriovenosa; cateterización; infección; dolor; supervivencia.

Evaluation of complications of arteriovenous fistula for hemodialysis according to the puncture technique

\begin{abstract}
Introduction: The arteriovenous fistula is the standard vascular access for the treatments in hemodialysis, punctured periodically three times per week. There are three types of puncture techniques: ladder, puncture area and buttonhole. Conventional techniques cause great morbidity in the medium and long term, however, a priori, the buttonhole technique is associated to the improvement of those problems.
\end{abstract}

Objective: To compare the buttonhole puncture technique with respect to conventional techniques for vascular access cannulation in the treatment of hemodialysis, depending on the degree of pain associated with the technique, associated infection rates and the repercussion on vascular access survival.

Material and Method: Narrative bibliographic review. Experimental and observational studies in English and Spanish published between 2010 and 2015 were included, comparing the conventional puncture technique versus the Buttonhole puncture technique. Initial 
selection by title and summary. The CASPe and STROBE scales were used for the methodological evaluation.

Results: 1.827 studies were identified, of which 13 were selected after the critical evaluation process. The heterogeneity of results does not allow to affirm improvements of any of the techniques analyzed in puncture pain or survival of the access, nevertheless, it is associated an increase of infectious events with the Buttonhole technique.

Conclusion: Any arguments have been found to ensure that Buttonhole technique can be considered as first choice due to the heterogeneity of the measurement methods of the reviewed studies.

KEYWORDS: hemodialysis; arteriovenous fistulae; catheterization; infection; pain; survival.

\section{Introducción}

La fístula arteriovenosa (FAV) autóloga es el acceso vascular de elección para los usuarios en tratamiento de hemodiálisis (HD) siendo el acceso permanente más seguro, de mayor duración, resultando de vital importancia para el usuario ${ }^{1,2}$. La reducción de las tasas de complicaciones y una mayor calidad dialítica fundamentan su elección en comparación a otros dispositivos.

El acceso a la FAV se realiza a través de la bipunción periódica, normalmente tres veces por semana dependiendo de las características del tratamiento de HD y el éxito de la canalización es fundamental para la realización de un tratamiento satisfactorio ${ }^{3}$. La técnica de punción influye directamente en la supervivencia del acceso vascular, el dolor, infección y otras complicaciones asociadas a la canulación. Existen tres tipos de técnicas de punción: en escalera, conocida en el ámbito anglosajón como "Rope Ladder", consiste en la distribución sucesiva de las punciones alrededor del recorrido venoso, para lo cual es necesario tener una FAV ya desarrollada; en área de punción, que consiste en distribuir las punciones sobre un área de 2-3 centímetros; y por último la Técnica del ojal o "buttonhole", que consiste en la punción repetida en el mismo punto, con el mismo ángulo y profundidad en cada sesión para la formación de un túnel subcutáneo que facilite sucesivas canalizaciones.

Históricamente, las técnicas de punción convencionales, en área de punción y en escalera, han sido las más empleadas 4 . Ambas son sencillas de ejecutar por los profesionales formados en el ámbito de diálisis, pero son cau- santes de gran morbilidad a medio y largo plazo asociada al acceso vascular, ya que ocasionan pequeños traumatismos que debilitan la pared del vaso sanguíneo y que forman un aneurisma en la zona de punción de la FAV 5 . A este proceso fisiopatológico se le conoce como remodelado iatrogénico ${ }^{6}$.

La técnica de buttonhole fue usada por primera vez en 1977 por el Dr. Twardowski para facilitar las punciones de aquellas FAV de difícil canulación o que disponían de un acceso venoso muy limitado ${ }^{5,7}$. Los resultados obtenidos pusieron de manifiesto una mejoría de los problemas asociados a la canulación convencional, como el dolor en la punción, aumento del tiempo de hemostasia, y el remodelado iatrogénic ${ }^{7}$. A pesar de los resultados, actualmente no existe consenso sobre los beneficios a priori del buttonhole, y el debate está abierto en relación a las complicaciones infecciosas durante la aplicación de esta técnica ${ }^{8}$. Por lo tanto, esta revisión de la literatura pretende comparar la técnica de punción de buttonhole con respecto a las técnicas convencionales para la canulación del acceso vascular en el tratamiento de Hemodiálisis, en función del grado de dolor asociado a la técnica, tasas de infección asociadas y la repercusión sobre la supervivencia del acceso vascular.

\section{Material y Método}

Revisión bibliográfica narrativa (Scoping Review) realizada siguiendo las directrices de la estrategia Prisma9.

\section{CRITERIOS DE ELEGIBILIDAD DE LOS ESTUDIOS:}

Tipos de estudio: Se seleccionaron estudios experimentales y observacionales que compararan las técnicas de punción convencional respecto a la técnica de punción Buttonhole.

Tipos de participantes: Adultos (edad por encima de 18 años) en tratamiento de HD crónico.

Tipos de medidas de resultado: Las medidas de resultado formaron parte de los criterios de inclusión de esta revisión y se agruparon en variables primarias y secundarias:

A) Variables primarias:

1. Dolor asociado a la punción

2. Infección asociada a la FAV

3. Supervivencia de la FAV 
B) Variable secundaria:

1. Formación de aneurisma en la FAV

\section{ESTRATEGIA DE BÚSQUEDA:}

Se utilizaron descriptores en castellano o inglés según la base de datos. Para ello se utilizaron las equivalencias del lenguaje estructurado DeCS (Descriptores en Ciencias de la Salud): Hemodiálisis, Cateterismo o Cateterización o Canulación, Fistula Arteriovenosa, Dolor, Evaluación del dolor, Supervivencia e Infección; y del vocabulario MeSH (en inglés, Medical Subjects Headings): Renal Dialysis, Catheterization, Cannulation, Arteriovenous fistula, Pain, Pain Measurement, Survival e Infection. Se efectuaron búsquedas en las siguientes bases de datos y recursos bibliográficos: Biblioteca Virtual en Salud (bvsalud-ISCIII), CUIDEN Plus, EBSCO-HOST (Medline y CINHAL), PubMed, OVID, ScienceDirect, SCOPUS y Web of science. Además, se realizó una búsqueda directa en otros recursos bibliográficos heterogéneos incluyendo selecciones de revistas sensibles a publicar el tema de estudio, hemerotecas o compendios de publicaciones como: JAMA Network (compendio de revistas de la American Medical Association) y CJASN (Clinical Journal of the American Society of Nephrology). Se limitó la búsqueda desde enero del 2010 hasta mayo del 2015.

\section{MÉTODOS DE LA REVISIÓN:}

Selección de los estudios: Se exploraron los títulos y resúmenes generados en las búsquedas. Se examinaron, además, listas de referencias de la bibliografía de los artículos revisados, y se recopilaron los resúmenes y posteriormente las publicaciones completas. Solo se incluyeron en la revisión los textos completos en inglés o en español.

Evaluación de la calidad metodológica de los estudios: Se utilizaron los criterios de calidad metodológica de CASP (Critical Appraisal Skills Programme) versión española ${ }^{10}$ para evaluar los estudios experimentales, y criterios de calidad metodológica de STROBE ${ }^{11}$ para estudios observacionales.

Extracción de datos: Se extrajeron características del estudio (país, año, diseño del estudio, tamaño de la muestra, y el estudio duración), las características del paciente (edad, sexo, modalidad de diálisis al inicio del estudio, y la edad de la FAV al inicio del estudio), tipo de técnica de canalización, y los principales resultados de la investigación.

\section{Resultados}

Resultados de Búsqueda: Se obtuvieron 1827 estudios, de los que 366 resultaron elegibles por su temática. Se eliminaron 202 estudios duplicados y 145 estudios que no cumplían los criterios de inclusión. Así fueron seleccionados 19 artículos para la evaluación de la calidad metodológica, de los que se eliminaron 6 en este proceso. Finalmente se seleccionaron 13 estudios para dar respuesta a los objetivos planteados, la figura 1 muestra el proceso de selección de los mismos.

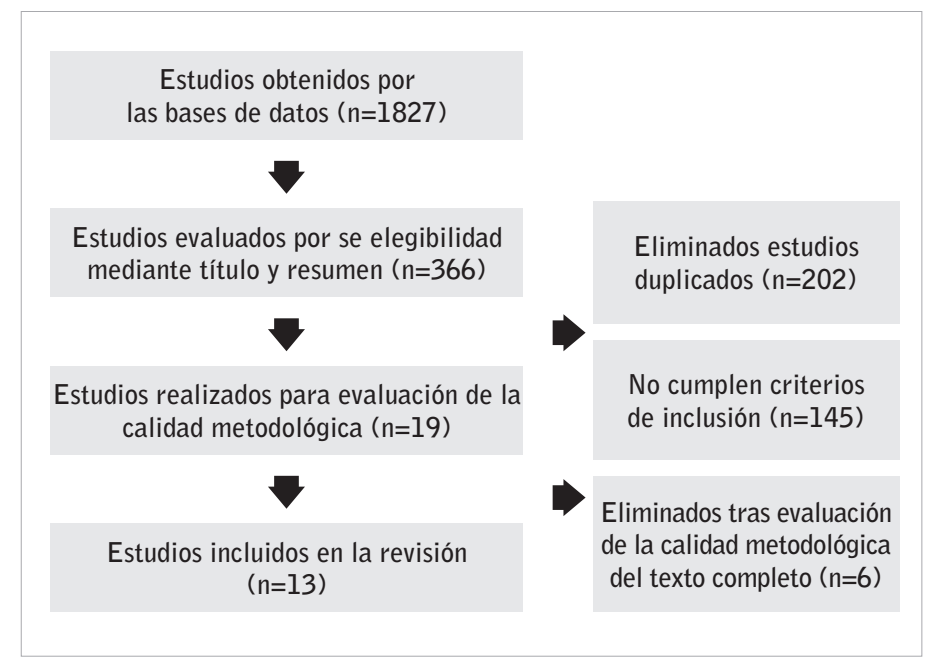

Figura 1. Diagrama de flujo documentando la inclusión de los estudios.

Características de los estudios y de los participantes: De los 13 estudios incluidos en la revisión (Tabla 1), hay 6 experimentales, de los cuales 5 son ensayos clínicos aleatorizados ${ }^{12-16}$ y 1 es un Clúster ${ }^{17}$, mientras que el resto de artículos seleccionados son observacionales, formados por 1 estudio de serie de casos retrospecti$\mathrm{va}^{18}, 2$ cohortes retrospectivas ${ }^{19,20}, 3$ cohortes prospectivas $^{21-23}$ y 1 estudio transversal ${ }^{24}$. 
Tabla 1. Características de los estudios y pacientes.

\begin{tabular}{|c|c|c|c|c|c|c|c|c|c|}
\hline Estudio & Diseño & País & $\begin{array}{l}\text { Criterio de } \\
\text { Resultado } \\
\text { Primario }\end{array}$ & $\begin{array}{c}\text { Tipo de } \\
\text { paciente y } \\
\text { modalidad }\end{array}$ & $\mathbf{N}$ & Edad Media & $\%$ Hombre & Edad FAV & $\begin{array}{l}\text { Tiempo } \\
\text { seguimiento } \\
\text { (media) }\end{array}$ \\
\hline $\begin{array}{l}\text { Aitken } \\
\text { (2013) }\end{array}$ & $\begin{array}{l}\text { Observ. } \\
\text { prospect. } \\
\text { transver. }\end{array}$ & Reino Unido & $\begin{array}{c}\text { Dolor en la } \\
\text { punción }\end{array}$ & $\begin{array}{l}\text { En centro } \\
\text { de HDC }\end{array}$ & $\begin{array}{c}449 \\
(209 \mathrm{BH} \\
238 \mathrm{PC} \\
2 \text { UP) }\end{array}$ & $60.5+/-0.72$ & $56.6 \%$ & 62 meses & NR \\
\hline $\begin{array}{l}\text { Chan } \\
\text { (2014) }\end{array}$ & $\begin{array}{l}\text { Cohorte } \\
\text { retrosp. }\end{array}$ & EEUU & $\begin{array}{c}\text { Superviven- } \\
\text { cia FAV }\end{array}$ & $\begin{array}{l}\text { En centro } \\
\text { de HDC }\end{array}$ & $\begin{array}{c}83 \\
(45 \mathrm{BH} 38 \\
\text { PC) }\end{array}$ & $\begin{array}{c}60.9+/-16.9 \\
\text { BH } \\
64.1+/-19.6 \\
\text { PC }\end{array}$ & $\begin{array}{l}64 \% \text { BH } \\
63 \% \text { PC }\end{array}$ & NR & 12 meses \\
\hline $\begin{array}{l}\text { Chow } \\
\text { (2011) }\end{array}$ & ECA & Australia & $\begin{array}{c}\text { Dolor en la } \\
\text { punción }\end{array}$ & $\begin{array}{c}\text { Domiciliario } \\
\text { y en centro } \\
\text { de HDC }\end{array}$ & $\begin{array}{c}69 \\
(34 \mathrm{BH} 35 \\
\text { PC) }\end{array}$ & NR & $\begin{array}{l}61.8 \% \mathrm{BH} \\
77.1 \% \mathrm{PC}\end{array}$ & NR & 6 meses \\
\hline$\underset{(2013)}{\text { Kim }}$ & $\begin{array}{l}\text { Estudio } \\
\text { Cluster }\end{array}$ & $\begin{array}{l}\text { Corea del } \\
\text { Sur }\end{array}$ & $\begin{array}{l}\text { Múltiples } \\
\text { resultados }\end{array}$ & $\begin{array}{l}\text { En centro } \\
\text { de HDC }\end{array}$ & 32 & 62.4 & $63 \%$ & 58.8 meses & $\begin{array}{l}16 \text { semanas } \\
\text { BH } \\
8 \text { semanas } \\
\text { PC }\end{array}$ \\
\hline $\begin{array}{l}\text { Labriola } \\
(2011)\end{array}$ & $\begin{array}{l}\text { Serie de } \\
\text { casos } \\
\text { retrosp. }\end{array}$ & Bélgica & $\begin{array}{l}\text { Proporción } \\
\text { de eventos } \\
\text { de Infección }\end{array}$ & $\begin{array}{l}\text { En centro } \\
\text { de HDC }\end{array}$ & 177 & $70.4+/-11.5$ & $65.8 \%$ & NR & 108 meses \\
\hline $\begin{array}{l}\text { Ludlow } \\
\text { (2010) }\end{array}$ & $\begin{array}{l}\text { Cohortes } \\
\text { prospect. }\end{array}$ & Canadá & $\begin{array}{l}\text { Múltiples } \\
\text { resultados }\end{array}$ & $\begin{array}{l}\text { En centro } \\
\text { de HDC }\end{array}$ & 29 & $\begin{array}{l}62.9 \mathrm{BH} \\
65.9 \mathrm{PC}\end{array}$ & $\begin{array}{l}62 \% \mathrm{BH} \\
58 \% \mathrm{PC}\end{array}$ & 32 meses & 3 meses \\
\hline $\begin{array}{l}\text { MacRae } \\
(2012)\end{array}$ & ECA & Canadá & $\begin{array}{l}\text { Múltiples } \\
\text { resultados }\end{array}$ & $\begin{array}{l}\text { En centro } \\
\text { de HDC }\end{array}$ & $\begin{array}{c}140 \\
(70 \mathrm{BH} 70 \\
\text { PC) }\end{array}$ & $\begin{array}{l}70.3 \mathrm{BH} \\
66.7 \mathrm{PC}\end{array}$ & $\begin{array}{l}72.9 \% \mathrm{BH} \\
65.7 \% \mathrm{PC}\end{array}$ & NR & 12 meses \\
\hline $\begin{array}{l}\text { MacRae } \\
(2014)\end{array}$ & ECA & Canadá & $\begin{array}{l}\text { Superviven- } \\
\text { cia FAV }\end{array}$ & $\begin{array}{c}\text { En centro } \\
\text { de HDC }\end{array}$ & $\begin{array}{c}139 \\
(70 \mathrm{BH} 69 \\
\text { PC) }\end{array}$ & $\begin{array}{l}70.2 \mathrm{BH} \\
66.1 \mathrm{PC}\end{array}$ & $\begin{array}{l}73 \% \text { BH } \\
67 \% \text { PC }\end{array}$ & $\begin{array}{c}\text { BH: } 26.2 \\
\text { meses } \\
\text { PC: } 31.9 \\
\text { meses }\end{array}$ & $\begin{array}{c}19.2 \text { meses } \\
\text { BH } \\
17.2 \text { meses } \\
\text { PC }\end{array}$ \\
\hline $\begin{array}{l}\text { Muir } \\
(2014)\end{array}$ & $\begin{array}{l}\text { Cohortes } \\
\text { retrosp. }\end{array}$ & Australia & $\begin{array}{l}\text { Infección } \\
\text { sistémica } \\
\text { asociada a } \\
\text { la FAV }\end{array}$ & $\begin{array}{c}\text { HDC } \\
\text { Domiciliaria }\end{array}$ & $\begin{array}{l}72 \mathrm{BH} \\
30 \mathrm{PC}\end{array}$ & 52 & 73 & NR & NR \\
\hline $\begin{array}{l}\text { Smyth } \\
\text { (2013) }\end{array}$ & $\begin{array}{l}\text { Cohorte } \\
\text { prospect. }\end{array}$ & Australia & $\begin{array}{l}\text { Múltiples } \\
\text { resultados }\end{array}$ & $\begin{array}{l}\text { En centro } \\
\text { de HDC }\end{array}$ & $\begin{array}{c}104 \\
(41 \mathrm{BH} 63 \\
\text { PC) }\end{array}$ & $\begin{array}{l}60 \mathrm{BH} \\
61 \mathrm{PC}\end{array}$ & $\begin{array}{l}34.1 \% \mathrm{BH} \\
55.6 \% \mathrm{PC}\end{array}$ & 31.2 meses & 12 semanas \\
\hline $\begin{array}{l}\text { Struthers } \\
(2010)\end{array}$ & ECA & Reino Unido & $\begin{array}{l}\text { Dolor en la } \\
\text { punción }\end{array}$ & $\begin{array}{c}\text { En centro } \\
\text { de HDC }\end{array}$ & $\begin{array}{c}56 \\
(28 \mathrm{BH} \\
28 \mathrm{PC})\end{array}$ & $\begin{array}{l}61 \mathrm{BH} \\
60 \mathrm{PC}\end{array}$ & $57.6 \%$ & $\begin{array}{l}28 \text { meses } \mathrm{BH} \\
25 \text { meses } \mathrm{PC}\end{array}$ & 6 meses \\
\hline
\end{tabular}




\begin{tabular}{|c|c|c|c|c|c|c|c|c|c|}
\hline Estudio & Diseño & País & $\begin{array}{c}\text { Criterio de } \\
\text { Resultado } \\
\text { Primario }\end{array}$ & $\begin{array}{c}\text { Tipo de } \\
\text { paciente y } \\
\text { modalidad }\end{array}$ & $\mathbf{N}$ & Edad Media & $\%$ Hombre & Edad FAV & $\begin{array}{l}\text { Tiempo } \\
\text { seguimiento } \\
\text { (media) }\end{array}$ \\
\hline $\begin{array}{l}\text { Van Loon } \\
(2010)\end{array}$ & $\begin{array}{l}\text { Cohorte } \\
\text { prospect. }\end{array}$ & Holanda & $\begin{array}{l}\text { Múltiples } \\
\text { resultados }\end{array}$ & $\begin{array}{l}\text { En centro } \\
\text { de HDC }\end{array}$ & $\begin{array}{c}145 \\
(75 \mathrm{BH} 70 \\
\text { PC) }\end{array}$ & $\begin{array}{l}67 \mathrm{BH} \\
65 \mathrm{PC}\end{array}$ & $\begin{array}{l}59 \% \text { BH } \\
67 \% \text { PC }\end{array}$ & $\begin{array}{l}44 \text { meses } \mathrm{BH} \\
31 \text { meses } \mathrm{PC}\end{array}$ & 9 meses \\
\hline $\begin{array}{l}\text { Vaux } \\
(2013)\end{array}$ & ECA & Reino Unido & $\begin{array}{c}\text { Superviven- } \\
\text { cia FAV }\end{array}$ & $\begin{array}{l}\text { En centro } \\
\text { de HDC }\end{array}$ & $\begin{array}{c}127 \\
(58 \mathrm{BH} 69 \\
\text { PC) }\end{array}$ & $\begin{array}{l}62 \mathrm{BH} \\
64 \mathrm{PC}\end{array}$ & $\begin{array}{l}67 \% \text { BH } \\
63 \% \text { PC }\end{array}$ & NR & 12 meses \\
\hline
\end{tabular}

FAV: Fistula Arteriovenosa HDC: Hemodiálisis Crónica PC: Punción convencional BH: Punción buttonhole

En 5 estudios no se especifica explícitamente el resultado primario de la investigación, abarcando múltiples resultados como el dolor en la punción, tasa de infección, tiempo de hemostasia, formación de aneurisma 0 supervivencia del acceso vascular. Los estudios con un único criterio de resultado fueron 8 , de los cuales 3 reportaron como criterio de resultado el dolor en la punción, 2 estudios se centraron en la tasa de infección en la FAV y 3 en la supervivencia de la misma. Todos los estudios incluyeron a pacientes realizando HD crónica, de los cuales un estudio incluyó solo a pacientes domiciliarios y uno combinó pacientes en centros de HD y en domicilio. El tamaño muestral osciló entre 29 y 449 pacientes. La edad media de los pacientes osciló entre 52 y 70.4 años. En 13 de los 14 estudios, la mayoría de los participantes fueron hombres con un rango entre $34.1 \%$ y $83 \%$. La edad media de la FAV al inicio del estudio se informó en 7 casos y osciló entre 25 y 62 meses. El seguimiento se notificó en 12 estudios, con un mínimo de 3 meses y un máximo de 108 meses.
Técnica de canulación: Existe gran heterogeneidad en la técnica de canulación empleada en cada estudio. Se emplean diferentes antisépticos cutáneos, en ocasiones se usan cremas anestésicas para aliviar el dolor en la punción y se emplean diferentes tipos y calibres de aguja. Además, falta información relevante para la comprensión de la técnica en su totalidad, como por ejemplo, el tiempo empleado para el desarrollo del túnel subcutáneo del buttonhole o el número de enfermeras que participaron.

Dolor en la canulación: En 9 estudios se informa sobre el dolor que percibe el paciente durante la canulación (Tabla 2), en 5 de los cuales se utilizó la escala Visual Analógica (EVA) $)^{14,15,21,24,25}$, en 3 estudios utilizaron la escala numérica de 10 puntos ${ }^{13,22,23}$ y en uno la escala de dolor de Wong-Baker ${ }^{16}$. La heterogeneidad de las formas de medida y los resultados no permiten esclarecer qué técnica de punción produce menos dolor. En este sentido, 2 estudios concluyen que la técnica de Buttonhole produce menor grado de dolor en la canulación, otros 3 señalan que la técnica de punción convencional es menos dolorosa y por último, en 4 no hay diferencias significativas entre ambas técnicas.

Tabla 2. Dolor en punción.

\begin{tabular}{|c|c|c|c|c|}
\hline Estudio & $\begin{array}{l}\text { Instrumento de medi- } \\
\text { ción del dolor }\end{array}$ & Buttonhole & Punción Convencional & p valor \\
\hline Aitken (2013) & EVA & 2.4 & 2.5 & $P=0.36$ \\
\hline Chow (2011) & $\begin{array}{l}\text { Escala del dolor de } \\
\text { Wong-Baker }\end{array}$ & $\begin{array}{c}\text { Inicio: } 0.81, \text { IC } 95 \% \\
0.41-1.20 \\
\text { Final seguimiento: } 0.56 \\
\text { IC } 95 \% 0.13-0.99\end{array}$ & $\begin{array}{c}\text { Inicio: } 0.81 \text {, IC } 95 \% \\
0.48-1.15 \\
\text { Final seguimiento: } 0.71 \\
\text { IC } 95 \% 0.34-1.09\end{array}$ & NR \\
\hline Kim (2013) & EVA & $\begin{array}{l}5.1+/-1.8 \mathrm{PV} \\
3.3+/-1.8 \mathrm{PA}\end{array}$ & $\begin{array}{c}6.3+/-1.3 \text { PV } \\
6.1+/- \text { PA }\end{array}$ & $\begin{array}{l}P=0.001 \\
P=0.001\end{array}$ \\
\hline
\end{tabular}


Tabla 2. Características de los estudios y pacientes. (continuación)

\begin{tabular}{|c|c|c|c|c|}
\hline Estudio & $\begin{array}{l}\text { Instrumento de medi- } \\
\text { ción del dolor }\end{array}$ & Buttonhole & Punción Convencional & p valor \\
\hline Ludlow (2010) & EVA & $\begin{array}{l}1.9+/-1.1 \text { PV } \\
1.7+/-0.8 \text { PA }\end{array}$ & $\begin{array}{l}2.6+/-1.4 \text { PV } \\
2.3+/-1.2 \text { PA }\end{array}$ & $\begin{array}{l}\mathrm{P}<0.01 \\
\mathrm{P}<0.002\end{array}$ \\
\hline MacRae (2012) & EVA & $\begin{array}{l}1^{\circ} \text { semana: } 1.6 \\
8^{\circ} \text { semana: } 1.5\end{array}$ & $\begin{array}{l}1^{\circ} \text { semana: } 1.5 \\
8^{\circ} \text { semana: } 1.2\end{array}$ & $\begin{array}{l}1^{\circ} \text { semana: } P=0.78 \\
8^{\circ} \text { semana: } P=0.57\end{array}$ \\
\hline Smyth (2013) & $\begin{array}{c}\text { Escala numérica de } 10 \\
\text { puntos }\end{array}$ & $1.90+/-1.069$ & $1.82+/-0934$ & $P=0.6$ \\
\hline Struthers (2010) & EVA & $\begin{array}{l}P: 3.0 \\
F: 2.5\end{array}$ & P y F: 1.0 & $P<0.01$ \\
\hline Van Loon (2010) & $\begin{array}{c}\text { Escala numérica de } 10 \\
\text { puntos }\end{array}$ & 1.6 & 1.0 & $P<0.001$ \\
\hline Vaux (2013) & $\begin{array}{c}\text { Escala numérica de } 10 \\
\text { puntos }\end{array}$ & 1.3 & 1.2 & $P=0.05$ \\
\hline
\end{tabular}

Tasa de infección: La asociación entre la tasa de infección local y/o sistémica y la técnica de canulación fue descrita en 9 estudios (Tabla 3). No existen criterios homogéneos para la medida de las tasas de infección. En
4 estudios se asoció significativamente el aumento de eventos infecciosos con la técnica de buttonhole, en 2 solo se obtuvieron eventos infecciosos para la técnica de Buttonhole y en 3 los resultados no fueron significativos.

Tabla 3. Tasa de infección.

\begin{tabular}{|c|c|c|c|c|c|}
\hline Estudio & Microorganismo & Complicación & Buttonhole & $\begin{array}{c}\text { Punción } \\
\text { Convencional }\end{array}$ & p valor \\
\hline Chan (2014) & NR & NR & $11 \%$ & $8 \%$ & $P=0.62$ \\
\hline Chow (2011) & $\begin{array}{l}\text { Klebsiella P y } \\
\text { Staphylococcus }\end{array}$ & $\begin{array}{l}\text { Infección local y } \\
\text { fiebre }\end{array}$ & $11.8 \%$ & $2.9 \%$ & $P=0.1$ \\
\hline Labriola (2011) & $\begin{array}{l}\text { S. Aureus } \\
\text { S. Epidermis }\end{array}$ & $\begin{array}{l}\text { Endocarditis y } \\
\text { Bacteriemia }\end{array}$ & $\begin{array}{l}3^{\circ} \text { Periodo: } 0.43 \\
\text { eventos / } 1000 \text { días } \\
4^{\circ} \text { Periodo: } 0.34 \\
\text { eventos / } 1000 \text { días }\end{array}$ & $\begin{array}{l}1^{\circ} \text { Periodo: } 0.17 \\
\text { eventos / } 1000 \text { días } \\
2^{\circ} \text { Periodo: } 0.11 \\
\text { eventos / } 1000 \text { días }\end{array}$ & $\begin{array}{c}P=0.003 \\
\text { (periodos } 1,2 \text { y } 3 \text { ) } \\
P=0.04 \\
\text { periodos } 3 \text { y } 4 \text { ) }\end{array}$ \\
\hline MacRae (2012) & $\begin{array}{l}\text { Staphylococcus } \\
\text { Aureus }\end{array}$ & $\begin{array}{c}\text { Bacteriemia y } \\
\text { absceso de la FAV }\end{array}$ & $\begin{array}{c}2 \text { eventos bacterie- } \\
\text { mia / } 12 \text { meses } \\
9 \text { eventos de absceso } \\
\text { / } 12 \text { meses }\end{array}$ & 0 eventos & $P=0.003$ \\
\hline Muir (2014) & NR & NR & $\begin{array}{l}\text { Medida 1: } 0.20 \\
\text { Medida 2: } 0.18 \\
\text { Medida 3: } 0.39\end{array}$ & $\begin{array}{l}\text { Medida 1: } 0.03 \\
\text { Medida 2: } 0.07 \\
\text { Medida 3: } 0.10\end{array}$ & $P<0.05$ \\
\hline Smyth (2013) & S. Aureus & Infección local & $\begin{array}{l}0.87 \text { eventos / } \\
1000 \text { días }\end{array}$ & $\begin{array}{l}0.76 \text { eventos / } \\
1000 \text { días }\end{array}$ & $P=0.9$ \\
\hline
\end{tabular}




\begin{tabular}{cccccc}
\multicolumn{1}{c}{ Estudio } & Microorganismo & Complicación & Buttonhole & $\begin{array}{c}\text { Punción } \\
\text { Convencional }\end{array}$ & p valor \\
Struthers (2010) & NR & NR & $\begin{array}{c}0.9 \text { eventos } / 1000 \\
\text { días }\end{array}$ & o eventos $/ 1000$ días & NR \\
Van Loon (2010) & $\begin{array}{c}\text { S. Aureus } \\
\text { Clostridium P. }\end{array}$ & NR & $5.3 \%$ & 0.001 \\
Vaux (2013) & S. Aureus & Bacteriemia & $\begin{array}{c}0.12 \text { eventos / } \\
1000 \text { días }\end{array}$ & $\begin{array}{c}0 \text { eventos / } \\
1000 \text { días }\end{array}$ \\
\hline
\end{tabular}

\section{*Labriola (2011).}

Periodo 1: consiste en canalizar a todos los pacientes con PC. Periodo 2: cambiar progresivamente a todos los pacientes a $\mathrm{BH}$. Periodo 3: canalización de $\mathrm{BH}$ antes de los talleres formativos. Periodo 4: canalización de BH después de los talleres formativos.

6 estudios informaron del microorganismo que provoca los eventos infecciosos, mostrándose al Staphylococcus Aureus como el agente más prevalente en los resultados de cultivo.
Supervivencia de la FAV: En 3 estudios se evalúa la supervivencia de la FAV asociada a la técnica de punción (Tabla 4), 2 de ellos como resultado principal. La medida de este resultado es heterogénea, en el estudio de Vaux et al. ${ }^{13)}$ se mide el porcentaje de fracaso del acceso, MacRae et al. ${ }^{12)}$ cuantifican el tiempo medio de funcionamiento de la FAV y en el estudio de Chan et al. ${ }^{20}$ se evalúa el porcentaje de accesos permeables realizando medidas a los 3, 6 y 12 meses. De ellos, el estudio realizado por Vaux et al..$^{13}$ aporta resultados estadísticamente significativos en favor de la técnica de Buttonhole.

Tabla 4. Supervivencia de la FAV.

\begin{tabular}{|c|c|c|c|c|}
\hline Estudio & Buttonhole & $\begin{array}{c}\text { Punción } \\
\text { Convencional }\end{array}$ & Notas & p valor \\
\hline Vaux (2013) & $0 \%$ & $13 \%$ & $\begin{array}{c}\text { El fracaso del acceso } \\
\text { se define como la FAV } \\
\text { que ya no se utilizada } \\
\text { para la realización } \\
\text { de HD }\end{array}$ & $P=0.005$ \\
\hline McRae (2014) & $\begin{array}{c}18.4 \text { meses (IC 10.9- } \\
32.7 \text { ) }\end{array}$ & $\begin{array}{l}16.0 \text { meses } \\
\text { (IC 10.6-29.3) }\end{array}$ & $\begin{array}{l}\text { La supervivencia del } \\
\text { acceso se define desde } \\
\text { el momento de inicio del } \\
\text { estudio hasta el abando- } \\
\text { no, muerte o cambio de } \\
\text { modalidad de HD al final } \\
\text { del seguimiento }\end{array}$ & $P=0.2$ \\
\hline Chan (2014) & $\begin{array}{c}3 \text { meses: } 86 \% \\
6 \text { meses: } 72\end{array}$ & $\begin{array}{c}3 \text { meses: } 89 \% \\
6 \text { meses: } 71\end{array}$ & $\begin{array}{l}\text { La disfunción del ac- } \\
\text { ceso se define como la } \\
\text { permeabilidad primaria } \\
\text { evaluada desde el inicio } \\
\text { del estudio adaptada a los } \\
\text { tiempos de seguimiento }\end{array}$ & $P=0.52$ \\
\hline
\end{tabular}

Formación de aneurisma: En 4 estudios se evalúa la formación de aneurisma en la FAV asociada a la técnica (Tabla 5), siempre como resultado secundario. En el estudio de Struthers et al. ${ }^{14}$ el aneurisma se define como una nueva dilatación del diámetro de la FAV medida de forma transversal. En los estudios de Vaux et al. ${ }^{13}$, Smyth et al. ${ }^{23}$ y Van Loon et al. ${ }^{22}$ se analiza el porcentaje de nuevos aneurismas desarrollados durante el estudio, obteniendo siempre resultados significativos a favor de la técnica de Buttonhole ${ }^{14,22,23}$. 
Tabla 5. Formación de aneurisma.

\begin{tabular}{|c|c|c|c|c|}
\hline Estudio & Buttonhole & $\begin{array}{c}\text { Punción } \\
\text { Convencional }\end{array}$ & Medición de aneurisma & $\mathrm{p}$ valor \\
\hline Smyth (2013) & $7.3 \%$ & $28.6 \%$ & $\begin{array}{l}\text { Nuevos aneurismas } \\
\text { desarrolladas en FAV en } \\
12 \text { semanas }\end{array}$ & $P=0.017$ \\
\hline Struthers (2010) & $1 \%+/-22 \%$ & $30 \%+/-7 \%$ & $\begin{array}{l}\text { Incrementado en el } \\
\text { diámetro del aneurisma } \\
\text { en } 6 \text { meses }\end{array}$ & $P<0.01$ \\
\hline Van Loon (2010) & $1 \%$ & $67 \%$ & $\begin{array}{c}\text { Nuevos aneurismas } \\
\text { desarrolladas en FAV en } \\
9 \text { meses }\end{array}$ & $P<0.01$ \\
\hline Vaux (2013) & $4 \%$ & $17 \%$ & $\begin{array}{c}\text { Nuevos aneurismas } \\
\text { desarrolladas en FAV en } \\
12 \text { meses }\end{array}$ & NR \\
\hline
\end{tabular}

\section{Discusión}

Esta revisión se plantea exponer de forma descriptiva una comparación entre las técnicas de punción disponibles para la canalización de la FAV. Este planteamiento no permite, sin embargo, determinar qué técnica produce menor grado de dolor, menores tasas de infección o cual se asocia a una mayor supervivencia del acceso vascular, debido a la heterogeneidad de las formas de medida entre los diferentes estudios.

En la actualidad, el criterio de inclusión para el Buttonhole se ha ampliado a todos los pacientes portadores de FAV, con respecto al que inicialmente se propuso. Su éxito depende en gran medida de la creación del túnel subcutáneo que facilitará las posteriores punciones ${ }^{26}$, siendo recomendable que la formación del túnel sea realizada por un único profesional(2), lo cual puede representar un desafío logístico para determinadas unidades de $\mathrm{HD}^{26,28}$. No obstante, existen experiencias en Canadá que han conseguido crear el túnel con la intervención de varios profesionales ${ }^{15}$. Además, para garantizar el éxito existe la necesidad de implementar un programa de formación dirigido al personal de enfermería que facilite la correcta realización del $\mathrm{BH}^{18,27}$. Estos han podido ser motivo por el cual no se ha conseguido extender definitivamente esta técnica. En un estudio realizado en 2013 en unidades de HD españolas4, la técnica de buttonhole fue empleada en un $6,7 \%$ de ellas. Estas cifras resultan escasas considerando que el $90,4 \%$ de los encuestados afirmaban conocer la técnica de buttonhole, aun- que aluden a "No haberse planteado la técnica" (29.4 $\%)$, "Existen problemas logísticos" (10.3\%) y "no saben cómo hacer la técnica" (7.9\%), apareciendo "la combinación de varias" técnicas en un $23.8 \%$. A pesar de esto, en los últimos años hemos podido observar un incremento progresivo en la utilización de la técnica de buttonhole en las unidades de HD, lo cual podría deberse a los teóricos beneficios que le otorgamos a esta técnica, como el menor grado de dolor en la punción, mayor facilidad para la autopunción y la punción de la FAV con tramos difíciles, reducción de la formación de hematomas y menor incidencia de formación de aneurismas en comparación con las técnicas de punción convencionales ${ }^{1,2}$. Otra posible razón podría ser la disponibilidad de ecógrafos Doppler, útiles para la evaluación y seguimiento de los accesos vasculares.

En relación al dolor secundario a la técnica de punción, no se encuentran resultados significativos a favor de ninguna técnica, independientemente de si los pacientes realizaban el tratamiento de $\mathrm{HD}$ en el domicilio 0 en su centro de referencia. Estos resultados resultan relevantes considerando que el alivio del dolor es asumido como un beneficio significativo de la técnica de buttonhole frente a la punción convencional. Serían necesarios estudios experimentales bien diseñados y de suficiente tamaño muestral para permitirnos dar respuesta al fenómeno del dolor asociado a la técnica de punción.

En cuanto a las tasas infección, la técnica de $\mathrm{BH}$ parece estar asociada a un incremento significativo de 
infecciones locales y sistémicas. A pesar de las estrictas medidas de asepsia introducidas en algunos a través de talleres educativos para el personal enfermero, se ha evidenciado una tasa de infección más elevada en la técnica de $\mathrm{BH}$ en comparación a la técnica convencional. En este sentido, la Guía de Práctica clínica de Kidney Disease Outcomes Quality Initiative (KDOQI) ${ }^{2}$ recomienda una técnica aséptica durante la técnica de canulación para disminuir las complicaciones infecciosas. El área de canulación deberá estar adecuadamente preparada para el procedimiento, se deberá realizar antisepsia cutánea, con una aplicación durante 30 segundos de Gluconato de Clorhexidina al $2 \%$ con alcohol al $70 \%$ o, en casos de alergias del paciente, Povidona iodada al $10 \%$ y eliminación de la costra, si se procede a realizar la técnica de $\mathrm{BH}^{2}$. El incremento de las tasas de infección puede ocasionar en el usuario renal un aumento significativo de la morbimortalidad, aumento de días de hospitalización, y en consecuencia, un aumento del gasto sanitario derivado al tratamiento ${ }^{29}$.

Con respecto a la supervivencia de la FAV, solo se identificaron 3 estudios ${ }^{12,13,20}$ que abordasen esta variable, sin encontrar resultados significativos respecto a que técnica de punción mejora la supervivencia del acceso vascular. Solamente el estudio de Vaux et al. ${ }^{13}$ encuentra que la técnica de $\mathrm{BH}$ mejora las expectativas de supervivencia de la FAV ( $p$ valor $=0,005$ ). El mayor número de punciones traumáticas y el mayor tiempo del endotelio expuesto a turbulencias de flujo ocasionaría mayores complicaciones respecto a la supervivencia de la FAV y al remodelado iatrogénico, que puede ser un mecanismo indirecto para la formación de estenosis y trombosis. Además, se produce una inflamación local que debilita progresivamente las paredes vasculares del endotelio. Por otro lado, hallamos resultados significativos en relación a la formación del aneurisma, los 4 estudios revisados $13,14,22,23$ evidencian que la técnica de $\mathrm{BH}$ ocasiona menor grado de dilatación de la FAV.

Una de las limitaciones de esta revisión es la baja calidad de los estudios en términos generales, atendiendo a la interpretación del nivel de evidencia de la Canadian Task Force on Preventive Health Care (CTFPHC). Son necesarios nuevos trabajos con diseños experimentales, unificación de las formas de medida de resultados y de la definición de las variables, con un seguimiento más prolongado en el tiempo, que permitan evaluar con mayor precisión los riesgos y beneficios de las diferentes técnicas.

Para finalizar podemos concluir que no se han hallado suficientes argumentos para asegurar que la técnica de buttonhole pueda ser considerada una técnica de primera elección para puncionar la FAV, dado que no se hallaron evidencias de mejora de indicadores de salud como el dolor en la punción y elevan el riesgo de complicaciones infecciosas respecto a las técnicas de punción convencional. Por esta razón, se recomienda seguir las guías de práctica clínica tomar una decisión multidisciplinar sobre la elección de la técnica de punción consensuando las preferencias del paciente junto con las características anatómicas de cada FAV.

No existe ningún conflicto de interés.

Agradecimientos a los compañeros de la unidad de diálisis del Hospital de Manacor.

Recibido: 5 diciembre 2016

Revisado: 25 enero 2017

Modificado: 5 febrero 2017

Aceptado: 15 febrero 2017

\section{Bibliografía}

1. Rodríguez J, González E, Gutiérrez J, Al. E. Guías de acceso vascular en hemodiálisis (Guías S.E.N.). Nefrologia. 2005;25(Supl 1):3-97.

2. Foundation National Kidney. KDOQI Clinical Practice Guidelines and Clinical Practice Recommendations for 2006 Updates: Hemodialysis Adequacy, Peritoneal Dialysis Adequacy and Vascular Access. Am J Kidney Dis. 2006; Supl 1(48):S1-322.

3. Atkar RK, MacRae JM. The buttonhole technique for fistula cannulation: pros and cons. Curr Opin Nephrol Hypertens. 2013;22(6):629-36. Available from: http://www.ncbi.nlm.nih.gov/pub$\mathrm{med} / 24076555$

4. Molina J, Amoros T. Análisis enfermero de la presencia de la Técnica del Ojal en las unidades de hemodiálisis españolas. Enferm Nefrol. 2013;16(2):93-8. Available from: http://scielo.isciii.es/pdf/enefro/v16n2/05_original4.pdf 
5. Twardowski Z, Kubara H. Different sites versus constant sites of needle insertion into arteriovenous fistulas for treatment by repeated dialysis. Dial Transplant. 1979;8(10):978-80.

6. Krönung G. Plastic deformation of Cimino fistula by repeated puncture. Dial Transplant. 1984;13:635-8.

7. Twardowski Z. Constant site (buttonhole) method of needle insertion for hemodialysis. Dial Transplant. 1995;24:559-76.

8. Gallieni M, Brenna I, Brunini F, Mezzina N, Pasho $\mathrm{S}$, Fornasieri A. Which cannulation technique for which patient. J Vasc Access. 2014;15(SUPPL. 7):85-90.

9. Urrútia G, Bonfill X. Declaración PRISMA: una propuesta para mejorar la publicación de revisiones sistemáticas y metaanálisis. Med Clin. 2010;135(11):507-11. Available from: http://linkinghub.elsevier.com/retrieve/pii/ S0025775310001454

10. Cabello J. Plantilla para ayudarte a entender un Ensayo Clínico. CASPe. Guías CASPe de lectura crítica de la literatura médica. CASPe. 2005;5-8.

11. Von Elm E, Altman G, Egger M, Pocock J, Gotzsche C, Vandenbroucke P. Declaración de la Iniciativa STROBE (Strengthening the Reporting of Observational studies in Epidemiology): directrices para la comunicación de estudios. Gac Sanit. 2008;22(2):144-50.

12. MacRae JM, Ahmed SB, Hemmelgarn BR. Arteriovenous fistula survival and needling technique: Long-term results from a randomized buttonhole trial. Am J Kidney Dis. 2014;63(4):636-42. Available from: http://dx.doi.org/10.1053/j. ajkd.2013.09.015

13. Vaux E, King J, Lloyd S, Moore J, Bailey L, Reading I, et al. Effect of buttonhole cannulation with a polycarbonate PEG on in-center hemodialysis fistula outcomes: a randomized controlled trial. Am J Kidney Dis. 2013;62(1):81-8. Available from: http://www.ncbi.nlm.nih.gov/pubmed/23473984

14. Struthers J, Allan A, Peel RK, Lambie SH. Buttonhole needling of ateriovenous fistulae: a randomized controlled trial. ASAIO J. 2010;56(4):319-22. Available from: http://www.ncbi.nlm.nih.gov/pub$\mathrm{med} / 20418768$
15. MacRae JM, Ahmed SB, Atkar R, Hemmelgarn $B R$. A randomized trial comparing buttonhole with rope ladder needling in conventional hemodialysis patients. Clin J Am Soc Nephrol. 2012; 7(10):1632-8. Available from: http://www. pubmedcentral.nih.gov/articlerender.

16. Chow J, Rayment G, San Miguel S, Gilbert M. A randomised controlled trial of buttonhole cannulation for the prevention of fistula access complications. J Ren Care. 2011;37(2):85-93.

17. Kim M, Kim H. Clinical effects of buttonhole cannulation method on hemodialysis patients. Hemodial Int. 2013;17(2):294-9.

18. Labriola L, Crott R, Desmet $C$, André $G$, Jadoul $M$. Infectious complications following conversion to buttonhole cannulation of native arteriovenous fistulas: a quality improvement report. Am J Kidney Dis. 2011;57(3):442-8.

19. Muir C a., Kotwal SS, Hawley CM, Polkinghorne K, Gallagher MP, Snelling P, et al. Buttonhole cannulation and clinical outcomes in a home hemodialysis cohort and systematic review. Clin J Am Soc Nephrol. 2014;9(5):110-9.

20. Chan MR, Shobande 0 , Vats $H$, Wakeen M, Meyer $X$, Bellingham $J$, et al. The effect of buttonhole cannulation vs. rope-ladder technique on hemodialysis access patency. Semin Dial. 2014;27(2):210-6.

21. Ludlow V. Buttonhole cannulation in hemodialysis: improved outcomes and increased expense--is it worth it? CANNT J. 2010;20(1):29-37. Available from: http://www.scopus.com/inward/ record.url?eid=2-s2.0-77952314776\&partnerI$\mathrm{D}=\mathrm{tZOt} \times 3 \mathrm{yl}$

22. van Loon MM, Goovaerts $T$, Kessels AGH, van der Sande FM, Tordoir JHM. Buttonhole needling of haemodialysis arteriovenous fistulae results in less complications and interventions compared to the rope-ladder technique. Nephrol Dial Transplant. 2010;25(1):225-30. Available from: http://www. ncbi.nlm.nih.gov/pubmed/19717827

23. Smyth W, Hartig V, Manickam V. Outcomes of buttonhole and rope-ladder cannulation techniques in a tropical renal service. J Ren Care. 2013;39(3):157-65. Available from: http://www. ncbi.nlm.nih.gov/pubmed/23799903 
24. Aitken E, McLellan A, Glen J, Serpell M, Mactier $R$, Clancy $M$. Pain resulting from arteriovenous fistulae: prevalence and impact. Clin Nephrol. 2013;80(5):328-33. Available from: http://www. ncbi.nlm.nih.gov/pubmed/23743153

25. Kim M-K, Kim H-S. Clinical effects of buttonhole cannulation method on hemodialysis patients. Hemodial Int. 2013;17(2):294-9. Available from: http://www.ncbi.nlm.nih.gov/pubmed/22998500

26. Besarab A. Access monitoring is worthwhile and valuable. Blood Purif. 2006;24:77-89.

27. Ball LK. The buttonhole technique for arteriovenous fistula cannulation. Nephrol Nurs J. 2006;33(3):299-304. Available from: http://www. ncbi.nlm.nih.gov/pubmed/16859201
28. Konner K, Nonnast-Daniel B, Ritz E. The arteriovenous fistula. J Am Soc Nephrol. 2003;14:1669-80.

29. Berenholtz SM, Lubomski LH, Weeks K, Goeschel CA, Marsteller JA, Pham JC, et al. Eliminating central line-associated bloodstream infections: a national patient safety imperative. Infect Control Hosp Epidemiol. 2014;35(1):56-62. Available from: http://www.scopus.com/inward/record. url?eid=2-s2.0-84890388265\&partnerID=tZ0tx3 ylfcgi?artid=3463206\&tool=pmcentrez\&renderty pe $=$ abstract 